DISCUSSION PAPER NO. 62

A STOCHASTIC MODEL OF PERSUASIVE COMMUNICATION

by

V. Balachandran and S. D. Deshmukh

November, 1973 



\title{
A STOCHASTIC MODEL OF PERSUASIVE COMMUNICATION
}

\begin{abstract}
This paper considers an information system involving a communicator who is interested in influencing a given decision maker's behavior through persuasive messages. For motivational reasons we first analyze a specific problem in a marketing context and then provide a general framework later. A source communicates a message repeatedly through a channel so as to persuade a subject to purchase a product. In this dynamic process a subject goes through exposure, comprehension, acceptance and purchase states. The state transitions are described by a semi-Markovian process which is nonstationary due to the subject's learning during revisits. Thus the probabilities of transitions to higher states and the times taken for such transitions improve with the number of transitions. The model facilitates analysis of transient and steady state behavior. Some measures of effectiveness of the communication are provided in order to compare different competing systems.
\end{abstract}





\section{A STOCHASTIC MODEL OF PERSUASIVE COMMUNICATION}

by

V. Balachandran and S. D. Deshmukh

\section{INTRODUCTION}

This paper considers an information system involving a communicator who is interested in influencing a given decision maker's behavior through persuasive messages. For motivational reasons we first analyze a specific problem in a marketing context and then provide a general framework later.

In media scheduling, a source repeatedly communicates a message through a channel to an audience with the final objective of persuading the audience to purchase a certain product. From the time a subject is exposed to the message till he makes a purchase he goes through a sequence of intermediate information processing states. His movement among these states through time is governed by a stochastic process. Thus it seems logical to represent and analyze such a persuasive communication process in the dynamic probabilistic framework. Further, in any given state, the subject not only decides probabilistically to move to some other state but also requires sorne random amount of time to make that decision. Moreover, it is realistic to expect that when he revisits a state a certain amount of learning has been acquired since his last visit and that this learning may lead to an improved chance of his moving closer to the final purchase state with a possible decrease in the time required to reach that state. Incorporation of these considerations leads to a non-stationary semi-Markovian model, which is described in Section 2.

Effectiveness of a communication depends on characteristics of the source, the channel and the audience. A professional expert or a trustworthy person may make a credible source yielding an effective communication (Hovland, Janis and Kelley [3], Jhonson and Watkins [7]). A video channel is likely to be more effective than an audio one. The demographic and personality characteristics 
of the audience affecting communication seem to have been studied more exhaustively by (McGuire [8]). A detailed review and excellent bibliography is provided by Sternthal [10]. In Section 3 we provide some quantitative measures of effectiveness of the source and the channel in communicating a message, so that, for an audience of given characteristics (assumed to be homogeneous and fixed) one may be able to compare abilities of different sources and channels in persuading the audience. One such measure is the average amount of time a subject requires before making a purchase, another criterion is the average amount of profit per unit time accruing due to such communication, while a third one is appropriately weighted average proportion of the audience found in each one of the information processing states per dollar invested in operating the system.

In the last section we give a general conceptual framework for analyzing persuasive communication as an explicit component of any decision-maker's problem.

\section{THE MODEL}

The model proposed here is based on the initial work of Hovland, Janis and Kelley [3] and McGuire [8], which was later applied to marketing by Howard and Sheth [6], Enge1, Kollat and Blackwel1 [2] and most recently presented by Sternthal [10] and Howard and Ostlund [5].

Given that the source emits a message through the channel it is necessary for communication that the subject be exposed to this message. Whether the subject is in this "exposure" state or not can be identified by observing his presence or absence. Due to existence of multiple stimuli he is usually selective 
in his exposure to each. For example, if his initial opinion about the message is favorable or if the message is novel and useful the probability of his being exposed to it is high. Similarly his education and self confidence also affect the selectivity of messages he chooses to receive.

Given that the subject is exposed, he either comprehends the message or becomes a candidate for further exposure. Between the exposure and the comprehension states the subject needs to go through the arousal and attention phases (see Sternthal [10]). Though good arousal and attention are necessary for comprehension, they are operationally difficult to measure, while comprehension can be measured by aided or unaided recall tests. Hence we will unify these into a single state to be called as "comprehension". Many factors such as the rate of communication and the degree of organization of the message affect comprehension.

Once the subject comprehends the message he either accepts or rejects it, which can be measured on an agreement-disagreement rating scale. Evidence suggests (McGuire [8]) that, if the subject rehearses thoughts consistent with the message received the message is likely to be accepted, while if he counterargues it then he becomes a candidate for further persuasion through exposure. If he accepts the message we will define him to be in the "acceptance" state. Given the subject in the acceptance state he may decide to purchase the product (and thus enter the "purchase" state) with certain probability; otherwise, he becomes a potential receiver of further communication.

Finally, even if he purchases the product at this time, he remains brand loyal only with a certain probability. If he changes his mind then he becomes a subject for further persuasion. Thus, it is not enough just to make sure that the subject accepts the message and makes a purchase, but this should be 
reinforced by continued communication.

The following notation will be used for the rest of the paper. Let the exposure, comprehension, acceptance and purchase states be indexed by $i=1,2,3$ and 4 respectively. Let $Y(t)$ denote the state of the subject at time $t \in[0, \infty)$ and let $J(n)$ be the state immediately after the $n \underline{t h}$ transition, $n=0,1,2, \ldots$. It is natural to assume that the subject starts at time 0 in the exposure state, so that $Y(0)=J(0)=1$. Denote by $X_{n}$ the time between the $(n-1)^{\text {st }}$ and the nth transition; for example, given that the subject has comprehended the message (i.e. $J(n-1)=2$ ), he takes a random amount of time $x_{n}$ to decide whether to accept it or not (i.e. $J(n)=3$ or 1 ). Let $M_{i}(t)$ denote the number of visits made to state $i$ in $[0, t]$ and let $M(t)$ be the vector $\left(M_{1}(t), \ldots M_{4}(t)\right)$. Similarly, let $N(n)=\left(N_{1}(n), \ldots, N_{4}(n)\right)$ be the vector of the number of visits $N_{i}(n)$ to state $i$ in $n$ transitions. (Note that $M(0)=N(0)=(1,0,0,0)$ and $\left.\sum_{i=1}^{4} N_{i}(n)=n+1\right)$.

The subject's learning process through time is reflected in the dependence of various probability distributions on $N(n)$ as described below.

$$
p_{i}\left(N_{i}\right)=\operatorname{Pr}\left[J(n+1)=i+1 \mid J(n)=i, N_{i}(n)=N_{i}\right], 1 \leq i \leq 3
$$

$$
\mathrm{p}_{4}\left(\mathrm{~N}_{4}\right)=\operatorname{Pr}\left[J(n+1)=4 \mid J(n)=4, N_{4}(n)=N_{4}\right]
$$

where $\mathrm{P}_{i}\left(\mathrm{~N}_{i}\right)$ is monotone non-decreasing in $\mathrm{N}_{i}$ (and hence has a limit as $\mathrm{N}_{\mathrm{i}} \rightarrow \infty$ ). Thus, in any state the probability of success (i.e. moving to a next higher state) increases with the number of visits made to that state, indicating the learning process induced by the persuasive communication. For $i=1, \ldots 4$, $\left(1-p_{i}\left(N_{i}\right)\right)$ is then the probability of going back to state 1 and thus being a candidate for exposure once again. The state transition probability matrix 
for the embedded Markov chain at the nth transition, given $N(n)=N$, can then be defined to be $\mathrm{P}(\mathrm{N})$ and its limit $\mathrm{P}$ as $\mathrm{N} \rightarrow \infty$ as follows.

$\begin{array}{cccc}1-\mathrm{p}_{1}\left(\mathrm{~N}_{1}\right) & \mathrm{p}_{1}\left(\mathrm{~N}_{1}\right) & 0 & 0 \\ 1-\mathrm{p}_{2}\left(\mathrm{~N}_{2}\right) & 0 & \mathrm{p}_{2}\left(\mathrm{~N}_{2}\right) & 0\end{array}$

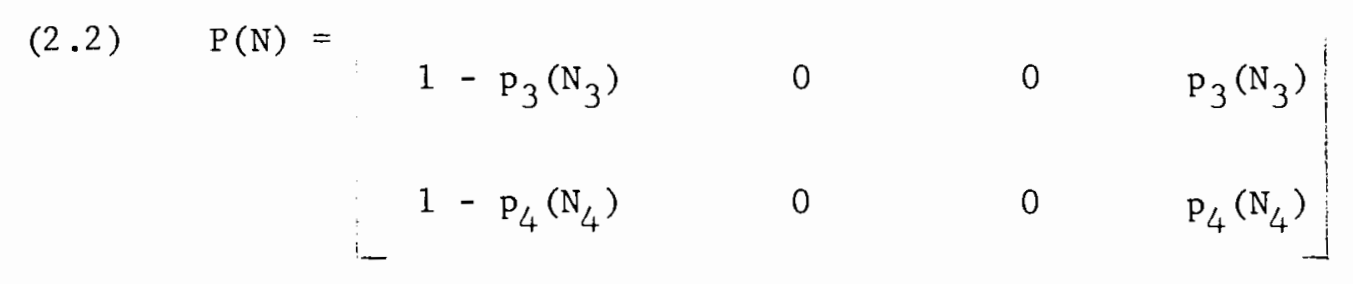

(2.3) $\quad \mathrm{p}_{i}=\lim _{\mathrm{N}_{\mathrm{i}} \rightarrow \infty} \mathrm{p}_{i}\left(\mathrm{~N}_{i}\right), 1 \leq i \leq 4, \quad \mathrm{P}=\lim _{\mathrm{N} \rightarrow \infty} \mathrm{P}(\mathrm{N})$

It is realistic and more interesting to assume that $p_{i}<1$ for all $i$, so that, even in the limit, communicatior is necessary to persuade the subject to make a purchase, i.e. the purchase state is not absorbing in $P$. Thus, as $N_{i} \rightarrow \infty$, every state stays recurrent and $M_{i}(t) \rightarrow \infty \quad 1 \leq i \leq 4$ as $t \rightarrow \infty$. In the limit the subject has learned as much as possible and there is no further learning, i.e. revisits to the states do not affect his decision process and hence the transition probabilities. The intertransition times have distributions defined as follows. Let

$$
\text { (2.4) } H_{i}\left(t \mid N_{i}\right)=\operatorname{Pr}\left[X_{n+1} \leq t \mid J(n)=i, N_{i}(n)=N_{i}\right], 1 \leq i \leq 4 .
$$

which is assumed to be monotone non-decreasing in $\mathrm{N}_{i}$ and hence has a limit as $\mathrm{N}_{\mathbf{i}} \rightarrow \infty$. Let 
(2.5) $\quad \mathrm{H}_{i}(t)=\lim _{\mathrm{N}_{i} \rightarrow \infty} \mathrm{H}_{i}\left(t \mid \mathrm{N}_{i}\right) \quad$ at all points of continuity of $\mathrm{H}_{i}(\cdot)$.

Thus the time required by the subject to make a decision decreases stochastically as his experience of being in that state accumulates. Let $\mu_{i}\left(N_{i}\right)$ be the corresponding expected amount of time, which is non-increasing in $\mathrm{N}_{i}$.

$$
\begin{aligned}
& \text { (2.6) } \mu_{i}\left(\mathrm{~N}_{i}\right)=\int_{0}^{\infty} t \mathrm{dH}_{i}\left(t \mid \mathrm{N}_{i}\right), 1 \leq i \leq 4 \\
& (2.7) \mu_{i}=\int_{0}^{\infty} t \mathrm{dH}_{i}(t)=\lim _{i} \mu_{i}\left(\mathrm{~N}_{i}\right), 1 \leq i \leq 4
\end{aligned}
$$

It is assumed that $\mu_{i}>0$, so that even after the subject is saturated with experience his decision process is not instantaneous. (Technically, this assumption is necessary to avoid the possibjlity of infinitely many transitions in a finite amount of time).

It should be noted that $\mathrm{p}_{1}\left(\mathrm{~N}_{1}\right), \mathrm{li}_{1}\left(\mathrm{t} \mid \mathrm{N}_{1}\right), \mathrm{p}_{1}$ and $\mathrm{H}_{1}(\mathrm{t})$ are more influenced by the source and channel than the other $\mathrm{P}_{i}\left(\mathrm{~N}_{i}\right), \mathrm{H}_{i}\left(t \mid \mathrm{N}_{i}\right)$ etc., which depend mainly on the audience characteristics. The first proposition examines the transient behavior of the embedded Markov chain. Here $\Pi(n)=\left(\Pi_{1}(n), \ldots \Pi_{4}(n)\right)$, where $\Pi_{i}(n)$ is the probability of the subject's being in state $i$ at the end of the nth transition.

Proposition 1 Given $\Pi(0)=\left(\begin{array}{llll}1 & 0 & 0 & 0\end{array}\right)$,

$$
\begin{aligned}
& E(N(0))=\left(\begin{array}{cccc}
1 & 0 & 0 & 0
\end{array}\right) \text { and } \\
& E(P(N(0))) \text { prespecified, }
\end{aligned}
$$

for $\mathrm{n}=1,2, \ldots$ the following recursion relations hold.

$$
\text { (2.8) } \quad \Pi(n)=\Pi(n-1) E[P(N(n-1))]
$$




$$
\begin{aligned}
& \text { (2.9) } E[N(n)]=E[N(n-1)]+\Pi(n) \\
& \text { (2.10) } E[P(N(n))]=E\left[P(N(n-1)]+\sum_{i=1}^{4}\left\{\Pi_{i}(n) E\left[P_{i}\left(N_{i}(n-1)+1\right)-P_{i}\left(N_{i}(n-1)\right] B_{i}\right\}\right.\right. \\
& \begin{array}{rrrr:rrrr}
-1 & 1 & 0 & 0 & 0 & 0 & 0 & 0 \\
0 & 0 & 0 & 0 & -1 & 0 & 1 & 0
\end{array} \\
& \text { where } \mathrm{B}_{1}=\begin{array}{llllllllll}
0 & 0 & 0 & 0
\end{array} \quad \mathrm{~B}_{2}=\begin{array}{llllll}
0 & 0 & 0 & 0
\end{array}
\end{aligned}
$$

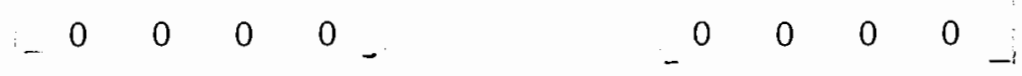

$$
\begin{aligned}
& \begin{array}{rrrrrrrr}
-0 & 0 & 0 & 0 & \cdots & 0 & 0 & 0
\end{array} \\
& \mathrm{~B}_{3}=\begin{array}{cccc:}
0 & 0 & 0 & 0 \\
-1 & 0 & 1 & 0
\end{array} \quad, \quad B_{4}=\begin{array}{llll}
0 & 0 & 0 & 0 \\
0 & 0 & 0 & 0
\end{array} \\
& \begin{array}{llllllll}
0 & 0 & 0 & 0 & -1 & 0 & 0 & 1
\end{array}
\end{aligned}
$$

proof: Given $N(n-1), \Pi(n-1)$ and $P(N(n-1))$ we have $\Pi(n)=\Pi(n-1) P(N(n-1))$ so that (2.8) follows. A1so, given $N(n-1), N(n)=N(n-1)+e_{i}$ with probability $\Pi(n-1) P^{i}(N(n-1))$ of visiting state $i$ next, where $\mathrm{P}^{i}\left(\right.$ ) denotes the $i$ th column of the matrix and $e_{i} i$ a vector of all 0 's except 1 in the ith place. Hence $E[N(n) \mid N(n-1)]=N(n-1)+\Gamma(n-1) P(N(n-1))$ giving $(2.9)$. Finally, $E[P(N(n)) \mid N(n-1)]=\sum_{i=1}^{4}\left[\Pi(n-1) P^{i}(N(n-1))\right] P\left(N(n-1)+e_{i}\right)$

$$
=\sum_{i=1}^{4} \prod_{i}(n) P\left(N(n-1)+e_{i}\right)
$$

$$
\begin{aligned}
& \text { while } P\left(N(n-1)+e_{i}\right)=P(N(n-1))+\left[P_{i}\left(N_{i}(n-1)+1\right)-P_{i}\left(N_{i}(n-1)\right)\right] B_{i} \\
& \text { yielding }(2.10) \quad \text { Q.E.D. }
\end{aligned}
$$

The communicator is interested in knowing the probability that the subject is in a specific state at some time in future given his state and the learning 
experience at present instant, say $T$. Define

$$
\text { (2.11) } Q_{i j}(t \mid N)=\operatorname{Prob}\left[Y(T+t)=j \mid Y(\tau)=i, M(T)=N, M\left(\tau^{-}\right)=N-e_{i}\right]
$$

Thus, $Q_{i j}(t \mid N)$ is the probability of the subject being in state $j t$ time units from now, given that he has just entered state $i$ and that his cumulative experience is summarized by the number of visits vector $N$. Since every state stays recurrent, we know that $\lim _{\tau \rightarrow \infty} M_{i}(\tau)=\infty$ with probability 1 , for all $i$. Then, with $M(\tau)=(\infty, \ldots \infty)$ we may define stationary probability in the long run by

$$
Q_{i j}(t)=\operatorname{Prob}[Y(t)=j \mid Y(0)=i]
$$

Finally, the steady state probability (which will be shown to exist) may be defined by

$$
\text { (2.13) } \mathrm{q}_{j}=Q_{i j}=\lim _{t \rightarrow \infty} Q_{i j}(t) \quad 1 \leq i \leq 4
$$

We shall use a convention that $i+1=4$ if $i=4$ and $\delta_{i j}= \begin{cases}1 & \text { if } i=j \\ 0 & \text { if } i \neq j\end{cases}$

\section{$\underline{\text { Proposition } 2}$}

$$
\begin{aligned}
& \text { (a) } Q_{i j}(t \mid N)=\int_{0}^{t}\left\{p_{i}\left(N_{i}\right) Q_{i+1, j}\left(t-x \mid N+e_{i+1}\right)\right. \\
& \left.+\left[1-p_{i}\left(N_{i}\right)\right] Q_{1 j}\left(t-x \mid N+e_{1}\right)\right\} d_{i}\left(x \mid N_{i}\right)+\delta_{i j}\left[1-H_{i}\left(t \mid N_{i}\right)\right] \\
& \text { (b) } Q_{i j}(t)=\int_{0}^{t}\left\{p_{i} Q_{i+1, j}(t-x)+\left[1-p_{i}\right] Q_{1 j}(t-x)\right\} \\
& d_{i}(x)+\delta_{i j}\left[1-H_{i}(t)\right]
\end{aligned}
$$


$(2.16)$

(c) $\mathrm{q}_{1}={ }^{\mu} 1 / \mathrm{D}, \quad \mathrm{q}_{2}=\mathrm{p}_{1}{ }^{\mu} / \mathrm{D}, \quad \mathrm{q}_{3}=\mathrm{p}_{1} \mathrm{p}_{2}{ }^{\mu} 3 / \mathrm{D}, \quad \mathrm{q}_{4}=\frac{\mathrm{p}_{1} \mathrm{p}_{2} \mathrm{p}_{3} \mu_{4}}{\left(1-\mathrm{p}_{4}\right) \mathrm{D}}$

$$
\text { where } D=\mu_{1}+p_{1} \mu_{2}+p_{1} p_{2} \mu_{3}+\frac{p_{1} p_{2} p_{3} \mu_{4}}{\left(1-p_{4}\right)}
$$

Proof: (a) and (b) follow by first conditioning on the time of the next transition out of state $i$ and the next state entered and then unconditioning. If $i=j$, the probability of not leaving $i$ by $t$ must be added to this.

To prove (c) define the Laplace-Stieltjes transform of a function $\mathrm{F}(\cdot)$ by $\tilde{F}(\mathrm{~s})=\int^{\infty} \mathrm{e}^{-s t} \mathrm{dF}(\mathrm{t})$. From (2.15) we get $\tilde{Q}_{i j}(s)=p_{i} \tilde{Q}_{i+1, j}(s) \tilde{H}_{i}(s)+\left[1-p_{i}\right] \tilde{Q}_{1 j}(s) \tilde{H}_{i}(s)+\delta_{i j}\left[1-\tilde{H}_{i}(s)\right]$.

Solving this set of equations and using the well known fact that

$$
\lim _{s \rightarrow 0} \tilde{F}(s)=\lim _{t \rightarrow \infty} F(t) \text {, yields (2.16), which is } q_{j}=\prod_{j} j_{j} \sum_{i=1}^{4} \prod_{i} \mu_{i} \text {, }
$$

where $\left(\Pi_{1}, \ldots \Pi_{4}\right)=\Pi$ is the vector of steady-state probabilities in the embedded Markov chain with transition matrix $P$ satisfying $\Pi=\Pi P$ and $\sum_{i=1}^{4} \Pi_{i}=1$. It turns out that

$$
\begin{aligned}
& \Pi_{1}=\left[1+p_{1} p_{2}+\left(p_{1} p_{2} p_{3}\right) /\left(1-p_{4}\right)\right]^{-1}, \Pi_{2}=p_{1} \Pi_{1}, \Pi_{3}=p_{1} p_{2} \Pi_{1} \text { and } \\
& \Pi_{4}=p_{1} p_{2} p_{3} \Pi_{1} 1 /\left(1-p_{4}\right) . \\
& \text { Q.E.D. }
\end{aligned}
$$

It is also of importance to the communicator to know the distribution of the time the subject takes to make a purchase since he is exposed. In general, 
define $r_{i j}(N)$ the random variable denoting the time at which the subject reaches state $j$ for the first time given that he has just entered state $i$ with his experience vector $N$. Let the corresponding distribution function and expectation be

$$
\begin{array}{ll}
\text { (2.17) } F_{i j}(t \mid N)=\operatorname{Prob}\left[r_{i j}(N) \leq t\right] & 1 \leq i, j \leq 4 \\
(2.18) \mu_{i j}(N)=E\left[r_{i j}(N)\right] & 1 \leq i, j \leq 4
\end{array}
$$

In steady state let $\mathrm{r}_{\mathbf{i} \mathbf{j}}, \mathrm{F}_{\mathbf{i j}}(t)$ and $\mu_{\mathbf{i} \mathbf{j}}$ be the corresponding values as $\mathrm{N}_{\mathrm{i}} \rightarrow \infty$ for a11 i.

\section{$\underline{\text { Proposition } 3}$}

(a) $F_{i, i+1}(t \mid N)=\int_{j}^{t}\left[1-p_{i}\left(N_{i}\right)\right] F_{1 j+1}\left(t-x \mid N+e_{1}\right) d H_{i}(x \mid N)+p_{i}\left(N_{i}\right) H_{i}(t \mid N)$

$$
\begin{aligned}
& F_{i, 1}(t \mid N)=\int_{0}^{t} p_{i}\left(N_{i}\right) F_{i+1,1}\left(t-x \mid N+e_{i+1}\right) d H_{i}(x \mid N)+\left[1-p_{i}\left(N_{i}\right)\right] H_{i}(t \mid N) \\
& F_{i j}(t \mid N)=\int_{0}^{t}\left[p_{i}\left(N_{i}\right) F_{i+1, j}\left(t-x \mid N+e_{i+1}\right)+\left[1-p_{i}\left(N_{i}\right)\right] F_{1 j}\left(t-x \mid N+e_{1}\right)\right] d H_{i}(x \mid N)
\end{aligned}
$$

$$
\text { if } j \neq i+1 \text { or } 1
$$

(b) $\mu_{i, i+1}(N)=\mu_{i}\left(N_{i}\right)+\left[1-p_{i}\left(N_{i}\right)\right] \mu_{1, i+1}\left(N+e_{1}\right)$

$$
\begin{gathered}
\mu_{i 1}(N)=\mu_{i}\left(N_{i}\right)+p_{i}\left(N_{i}\right) \mu_{i+1}\left(N+e_{i+1}\right) \\
\mu_{i j}(N)=\mu_{i}\left(N_{i}\right)+\left[1-p_{i}\left(N_{i}\right)\right]_{i j}\left(N+e_{1}\right)+p_{i}\left(N_{i}\right) \\
\mu_{i+1, j}\left(N+e_{i+1}\right) \text { if } j \neq i+1 \text { or } 1
\end{gathered}
$$

(c) $F_{i j}(t)$ and $\mu_{i j}$ satisfy expressions (2.16) and (2.17) respectively. 
Proof: (a) The event of going from $i$ to $i+1$ for the first time in time $t$ can be realized by either making the next transition to $i+1$ at time $t$ or by making the next transition to state 1 at time $x$ and then going to $(i+1)$ for the first time in the remaining time $(t-x)$. Similar argument yields the other expressions in (2.19)

(b) The average time required to go from i to $(i+1)$ is the sum of the average time spent in $i$ before making a transition and the average time required to go from the new state to $i+1$ for the first time. Other equations in $(2.20)$ follow similarly.

(c) follows similarly.

Q.E.D.

Solving $(2.20)$ yields $\mu_{14}$, the average amount of time the subject requires in the long run to make a purchase since being exposed to the message

$$
\text { (2.21) } \mu_{14}=\frac{\mu_{1}}{\mathrm{p}_{1} \mathrm{p}_{2} \mathrm{p}_{3}}+\frac{\mu_{2}}{\mathrm{p}_{2} \mathrm{p}_{3}}+\frac{\mu_{3}}{\mathrm{p}_{3}}
$$

Similarly, it can be shown that the average time between successive purchases $\mu_{44}$ and the average time between the subject being available for exposure $\mu_{11}$ are given by

$$
\begin{aligned}
& \text { (2.22) } \mu_{44}=\sum_{i=1}^{4} \prod_{i} \mu_{i} / \Pi_{4}=\mu_{4}+\left(1-p_{4}\right) \mu_{14} \\
& (2.23) \mu_{11}=\sum_{i=1}^{4} \Pi_{i} \mu_{i / \Pi_{1}}=\mu_{1}+p_{1} \omega_{2}+p_{1} p_{2} \mu_{3}+\frac{p_{1} p_{2} p_{3}}{\left(1-p_{4}\right)} \mu_{4}
\end{aligned}
$$

where $\Pi_{i}$ 's are as in $(2.16)$. 
In the above definitions of $P(N), H_{i}\left(t \mid N_{i}\right), Q_{i j}(t \mid N)$ and $F_{i j}(t \mid N)$ we have incorporated learning through the subject's experience descriled by $N$ to influence his behavior pattern. Such a behavior pattern has a transient and a steady state component, where the latter has been analyzed above.

In the short run we are interested in the transient behavior, which can be studied using geometric transform methods. For example, from (2.14) upon taking the Laplace-Stieltjes transform we get

$$
\begin{aligned}
& \text { (2.24) } \tilde{Q}_{i j}\left(s \mid N_{1}, \ldots N_{4}\right)=\left[p_{i}\left(N_{i}\right) \tilde{Q}_{i+1, j}\left(s \mid N_{1}, \ldots N_{i}+1, \ldots N_{4}\right)\right. \\
& \left.+\left[1-p_{i}\left(N_{i}\right)\right] \tilde{Q}_{1 j}\left(s \mid N_{1}+1, \ldots N_{2}, \ldots N_{4}\right)-\delta_{i j}\right] \tilde{H}_{i}\left(s \mid N_{i}\right)
\end{aligned}
$$

To analyze the behavior of $\tilde{Q}_{i j}\left(s_{i} N_{1}, \ldots N_{4}\right)$ as $N_{i}(i=1, \ldots 4)$ increase in integral steps we may define its joint $z$-transform (Howard [4])

$Q_{i j}\left(s \mid z_{1}, z_{2}, z_{3}, z_{4}\right)=\overbrace{N_{4}=0}^{\infty} \cdots \sum_{N_{1}=0}^{\infty} \tilde{Q}_{i j}\left(s \mid N_{1}, \ldots N_{4}\right) z_{1}^{N_{1}}{ }^{N_{2}}{ }_{2} z_{3}{ }^{N_{3}} z_{4}^{N_{4}} \quad$ where $\left|z_{i}\right|<1$, $i=1, \ldots 4$. Such a transform facilitates analytical manipulation resulting in explicit identification of the total behavior as a sum of the transient and steady state components. (For an excellent discussion and examples see Howard [4].) However, inspection shows that taking transforms in (2.24) requires definition of transforms for products of general functions, for which usual techniques do not apply. Thus in alsence of the closed form analytical solution of the transient behavior we resort to simulation techniques.

The recursive computation of the probabilities $\left\{Q_{i j}(t \mid N)\right\}$, for example, can be carried out utilizing the following algorithm.

Step 0: (Initialization). Let $Q_{i j}(0 \mid N)=\delta_{i j}$ for all $N$. Store all $P_{i}\left(N_{i}\right)$ and the inverse of $\mathrm{H}_{i}\left(\left.\cdot\right|_{i}\right)$ for a11 $\mathrm{N}_{i}=0,1, \ldots \mathrm{N}_{\max }$ and $i=1, \ldots 4$. 
(Such inverse functions for standard distributions are available in many simulation packages.) Let $\mathrm{L}=0, \mathrm{~T}=0$.

Step 1: Let $\mathrm{N}=\left(\begin{array}{llll}1 & 0 & 0 & 0\end{array}\right) \cdot \mathrm{T}=\mathrm{T}+\Delta \mathrm{T}$. (where $\Delta \mathrm{T}$ is a small discrete time step.) If $T>\operatorname{Tmax}$ then go to step 7 .

Step 2: Starting with $i=1$ and increasing it by 1 till $i=4$ repeat the following steps.

Step 3: Given $i$, repeat the following steps for $\mathrm{N}_{i}=0,1, \ldots \mathrm{N}_{\mathrm{Max}}$.

Step 4: For each $j=1,2, \ldots 4$, repeat the following steps.

Step 5: Generate a random number $r \in[0,1]$ and find the corresponding deviate $x=H_{i}^{-1}\left(r \mid N_{i}\right)$. If $x \geq T$ then set $Q_{i j}(T \mid N)=\delta_{i j}$ for all $j$. Let $\mathrm{N}_{\mathrm{i}}=\mathrm{N}_{\mathrm{i}}+1$ and repeat step 5 .

Step 6: Since $x<T$, use the same $r$ to check if $r \leq p_{i}\left(N_{i}\right)$. If so then set $k=i+1$, else set $k=i$. Then

$Q_{i j}(t \mid N)=Q_{k j}\left(T-\left[\frac{T-x}{\Delta T}\right] \Delta T \mid N+e_{k}\right)$, where $[y]$ denotes the integral part of $y$. If all i's. $j$ 's and $N_{i}$ 's have been considered, then $\mathrm{T}=\mathrm{T}+\Delta \mathrm{T}$. If $\mathrm{T}>\operatorname{Tmax}$ go to step 7 otherwise go to step 2 . E1se go to the relevant step 3 or 4 .

Step 7: Set $\mathrm{T}=0, \mathrm{~L}=\mathrm{L}+1$. If $\mathrm{L}=\mathrm{Lmax}$, go to step 8 . Else go to 1 . Step 8: For $i, j=1, \ldots 4, T=0, \ldots T_{\text {Max }}: N=0, \ldots N_{M a x}$ add $Q_{i j}(T \mid N)$ for a11 $L=1, \ldots, L_{\operatorname{Max}}$ and divide the total by $L_{\max }$ to give $Q_{i j}\left(T^{\prime} N\right)$.

Similar algorithms can be developed for computing other probabilities to yield the entire transient behavior. The next section considers the normative implications of the model for the communicator taking into consideration the 
long run steady state behavior of the audience. The transient behavior output of the simulation could as well be utilized in the next section to yield similar results for the transient case.

\section{OPTIMAL SYSTEM DESIGN}

Designing an effective communication system involves selecting an optimal combination of a source and a channel so as to maximize some measure of communication effectiveness in persuading the audience of given characteristics. In the previous section it was assumed that the message is shown continuously through time. However, it is more realistic to assume that the message is shown intermittently. Let $\mathrm{T}_{1}$ be the time for which the system is to be "on" and let $\mathrm{T}_{2}$ be the consecutive time for which it is to be "off", together the total duration $\left(\mathrm{T}_{1}+\mathrm{T}_{2}\right)$ will be cal1ed a cycle length. Let $a=\frac{\mathrm{T}_{1}}{\mathrm{~T}_{1}+\mathrm{T}_{2}}$ be the proportion of time the system is to be on; it is the system availability. Suppose $C_{1}$ is the cost of showing the message per unit time and $C_{2}$ is the cost of maintaining the system when it is off per unit time, $C_{1}>C_{2} \geq 0$. Define $r$ to be the profit per unit time when the subject makes a purchase.

Given a source, a channel and an audience, the objective is to determine an optimal utilization of this communication system by determining $\alpha^{*}$ so as to maximize the net profit per unit time in the long run given by

$$
\text { (3.1) } \mathrm{r}_{4}-\frac{\mathrm{C}_{1} \mathrm{~T}_{1}+\mathrm{C}_{2} \mathrm{~T}_{2}}{\mathrm{~T}_{1}+\mathrm{T}_{2}}
$$

where $\bar{\Pi}_{4}$ is the expected proportion of time the subject makes a purchase.

\section{Proposition 4}

The optimal proportion of time the message should be shown is given by 


$$
\begin{aligned}
& \alpha^{*}=\left[\sqrt{\frac{r p_{1} p_{2} p_{3}}{\left(1-p_{4}\right)\left(c_{1}-c_{2}\right)}-1}\right]\left[\left[p_{1}+p_{1} p_{2}+\frac{p_{1} p_{2} p_{3}}{\left(1-p_{4}\right)}\right],\right. \\
& \text { where } 0 \leq \alpha^{*} \leq 1
\end{aligned}
$$

proof: In the long run, given the system is on (which happens with probability $\left.x=\frac{\mathrm{T}_{1}}{\mathrm{~T}_{1}+\mathrm{T}_{2}}\right)$ the transition matrix is identical with the one in (2.3). However, the first row of the matrix will be changed to $\left(\begin{array}{llll}1 & 0 & 0 & 0\end{array}\right)$ if the system is off (which happens with probability $1-\propto)$. Thus the long run transition matrix for such an on-off system has the first row $\left(\begin{array}{lllll}1-\alpha p_{1} & \alpha p_{1} & c & 0\end{array}\right)$, the remaining rows being the same as in (2.3). The corresponding steady state probability $\bar{\Pi}_{4}$ is given by

$$
\begin{aligned}
& \text { (3.3) } \bar{\Pi}_{4}=\frac{\approx p_{1} p_{2} p_{3}}{\left(1-p_{4}\right)}\left\{1 \ldots\left[p_{1}+p_{1} p_{2}+\frac{p_{1} p_{2} p_{3}}{\left(1-p_{4}\right)}\right]\right\}^{-1} \\
& =\frac{A \alpha}{1+B \alpha} \text {, where } \\
& \text { (3.4) } \quad A=\frac{p_{1} p_{2} p_{3}}{\left(1-p_{4}\right)}, \quad B=p_{1}+p_{1} p_{2}+\frac{p_{1} p_{2} p_{3}}{\left(1-p_{4}\right)}, \quad B \geq A \geq 0
\end{aligned}
$$

Then (3.1) becomes

$$
\text { (3.5) } \quad R(\alpha)=\frac{r A \alpha}{1+B \alpha}-C_{1} \alpha-c_{2}(1-\alpha)
$$

which is concave in $\propto$ and is maximized when 


$$
\text { (3.6) } a=\alpha^{*}=\frac{1}{B}\left[\sqrt{\frac{r A}{\left(C_{1}-C_{2}\right)}-1}\right] \text {, which is (3.2) }
$$

Q.E.D.

Note that the communication source and channel determine A, B, (through $\left.\mathrm{p}_{i}^{\prime} \mathrm{s}\right), \mathrm{C}_{1}$ and $\mathrm{C}_{2}$. If $\mathrm{rA}<\left(\mathrm{C}_{1}-\mathrm{C}_{2}\right)$ we define $a^{*}=0$; in that case the cost of showing the message is prohibitive in relation to the profit resulting from sales, $\mathrm{C}_{2}$ being the resulting cost. If, on the other hand, rA $>\left(C_{1}-C_{2}\right)(B+1)^{2}$ we define $\alpha^{*}=1$, yielding the continuous communication process considered in section 2 with the average profit rate of $\left(\frac{r A}{1+B}-C_{1}\right)$. The set of communication sources and channels yielding $0<\alpha^{*}<1$ given by (3.2) is non empty since $B>0$. Thus operating a given source and a channe1 optimally so as to persuade a given audience yields the net long run return per unit time

$$
\text { (3.7) } R\left(\alpha^{*}\right)=\frac{1}{B}\left[C_{1}-C_{2}(B+1)+1-\sqrt{\frac{\left(C_{1}-C_{2}\right)}{r A}}(1+r A)\right]
$$

The next problem of interest is to choose an optimal source and a channel for a given audience so as to maximize the average long run net return per unit time. Let $\&$ be the set of all available sources and let $C$ be the set of al1 available channels communicator can choose from. Now all the channels may not be compatible with all the sources, (due to, for example, language or coding difficulties), hence, for any source $s \in \mathcal{\&}$, let $\Gamma(s) \subset \subset$ be the set of compatible channels, thus $\Omega=\{(s, c): s \in \rho, c \in \Gamma(s)\}$ is the feasible set of the communication systems to choose from. For each $\omega=(s, c) \varepsilon \Omega$ 
we have the cost of procuring (or leasing) that system given by $K(\omega)$ with an upper limit on total budget as L. Also associated with each $\omega \in \Omega$ we have the operating and maintenance costs $C_{1}(\omega)$ and $C_{2}(\omega)$ respectively and constants $A(\omega)$ and $B(w)$ as in $(3.4)$. Let $\alpha *(\omega)$ be the optimal proportion of time the s;.stem $\omega$ is to be on as given by (3.6). Then the problem is to choose an optimal system $\omega^{*}=\left(s^{*}, c^{*}\right)$ which solves

$$
\begin{aligned}
\operatorname{Max} R\left(\alpha^{*}(\mathrm{~s}, \mathrm{c})\right) & \\
\text { (3.8) } \mathrm{s.t.} \mathrm{K}(\mathrm{s}, \mathrm{c}) & \leq \mathrm{L} \\
\mathrm{s} & \in \mathscr{\varrho} \\
\mathrm{c} & \in \Gamma(\mathrm{s}) \subset \mathrm{c}
\end{aligned}
$$

This yields a conceptual formulation of the problem of optimal design, which may be solved when the functions involved are known. A heuristic sequential procedure would be to choose for each source $s \in \mathscr{\&}$ an optimal channel $\mathrm{c}^{*}(\mathrm{~s}) \in \Gamma(\mathrm{s})$ so as to yield a maximum $\mathrm{R}\left(\alpha^{*}(\mathrm{~s}, \mathrm{c} *(\mathrm{~s}))\right)$ subject to $\mathrm{K}(\mathrm{s}, \mathrm{c} *(\mathrm{~s})) \leq \mathrm{L}$, and then to choose the best source $s^{*}$ which yields a maximum $\mathrm{R}\left(\alpha^{*}\left(\mathrm{~s}^{*}, \mathrm{c}^{*}\left(\mathrm{~s}^{*}\right)\right)\right)$; then the total optimal system is $\omega^{*}=\left(s^{*}, c^{*}\left(s^{*}\right)\right)$.

Another possible measure of effectiveness of the communication system is the average amount of time a subject requires to make a purchase since being exposed to the message, as given by $\psi_{14}$ in (2.21). Since the decision times $\mu_{1}, \mu_{2}, \mu_{3}$ and $\mu_{4}$ are dependent mainly on the audience characteristics, the source and the channel influence $\mu_{14}$ through $p_{1}, p_{2}, p_{3}$. For simplicity assume that for any values of $\left(\mathrm{p}_{1}, \mathrm{p}_{2}, \mathrm{p}_{3}\right)$ there exists a feasible communication 
system $\omega \in \Omega$ giving those probabilities. If there are more than one such (1) we select the least expensive system. Thus selecting a system is equivalent to choosing $\left(p_{1}, p_{2}, p_{3}\right)$, which solves the following

$$
\begin{gathered}
\text { Min. }{ }_{{ }_{1}} \mathrm{p}_{1}^{-1} \mathrm{p}_{2}^{-1} \mathrm{p}_{3}^{-1}+\omega_{2} \mathrm{p}_{2}^{-1} \mathrm{p}_{3}^{-1}+\omega_{3} \mathrm{p}_{3}^{-1} \\
\text { s. t. } 0 \leq \mathrm{p}_{1} \leq 1 \\
0 \leq \mathrm{p}_{2} \leq 1 \\
0 \leq \mathrm{p}_{3} \leq 1 \\
\frac{\mathrm{k}\left(\mathrm{p}_{1}, \mathrm{p}_{2}, \mathrm{p}_{3}\right)}{\mathrm{L}} \leq 1
\end{gathered}
$$

where $K\left(P_{1}, P_{2}, P_{3}\right)=K(w), \omega$ being the corresponding system. Assume $\lim _{\mathrm{im}} \mathrm{K}\left(\mathrm{p}_{1}, \mathrm{p}_{2}, \mathrm{p}_{3}\right)=\infty$ and $\mathrm{K}\left(\mathrm{p}_{1}, \mathrm{p}_{2}, \mathrm{p}_{3}\right)$ is increasing in $\mathrm{p}_{i}$ for each $i$, thus $p_{i} \rightarrow 1$

implying that a perfect persuasion is prohibitively expensive. If the $\mathrm{K}$ function is a polynomial with positive coefficients (or a posynomial) or can be approximated by a posynomial, then (3.9) becomes a geometric program (see Duffin et. al [1]). This program can be solved utilizing the geometric programming algorithm to yield the optimal solution $\left(\mathrm{p}_{1}^{*}, \mathrm{p}_{2}^{*}, \mathrm{p}_{3}^{*}\right)$ and thus the corresponding system $\omega^{*}$ which minimizes the expected time between exposure and purchase states. Further from the normality condition (see Duffin et. al [1] p. 79) the three dual variables corresponding to the three terms of the objective function give us the relative importance of different $p_{i}$ 's in the optimal $14^{\circ}$ It turns out that it is better to invest resources to increase $\mathrm{p}_{3}$ relative to $\mathrm{P}_{2}$ or $\mathrm{p}_{1}$ and to increase $\mathrm{p}_{2}$ relative to $\mathrm{p}_{1}$. This means, 
for example, that it is more rewarding to increase the probability of the subject's accepting the message given that he has comprehended it than that of his comprehending the message upon exposure.

In the same spirit we may consider the problem of choosing the system so as to minimize $\mu_{44}$, the long run average amount of time between successive purchases. From (2.22) it is seen that the objective function, $\mu_{4}+\left(1-p_{4}\right) \mu_{14}$, is a linear transformation of the one in (3.9). The new problem of determining optimal $\left(\mathrm{p}_{1}, \mathrm{p}_{2}, \mathrm{p}_{3}, \mathrm{p}_{4}\right)$ is then similar to (3.9), so that it will yield similar conclusions as above, though the optimal solution may be different.

Another general criterion for comparing cost-effectiveness of different communication systems would be the expected utility per dollar invested. In any ceneral persuasive communication process the communicator may value not only the audience in the final state 4 but also those in the intermediate states of acceptance and comprehension as well. Let wi be the utility to the communicator per unit time of findirg the subject in state $i(i=1, \ldots 4)$. (In general, $\mathrm{U}_{1} \leq \mathrm{U}_{2} \leq \mathrm{U}_{3} \leq \mathrm{U}_{4} \cdot$ ) Recall from (2.13) that $\mathrm{q}_{\mathrm{i}}$ is the probability of finding the subject in state $i$ in the long run. Invoking the earlier definitions of $c_{1}, c_{2}$ and $\alpha$ and $(2.16)$ we wish to maximize the expected utility per unit cost of operation given by

$$
\text { (3.10) } U=\frac{\mu_{1} u_{1}+\alpha p_{1} \mu_{2} u_{2}+\alpha p_{1} p_{2} \mu_{3} u_{3}+\left(\alpha p_{1} p_{2} p_{3} \mu_{4} \mu_{4}\right) /\left(1-p_{4}\right)}{\left[\mu \mu_{1}+\alpha p_{1} \mu_{2}+\alpha p_{1} p_{2} \omega_{3}+\left(\alpha p_{1} p_{2} p_{3} \mu_{4}\right) /\left(1-p_{4}\right)\right]\left[c_{1} \alpha+c_{2}(1-\alpha)\right]}
$$

Evaluating $U$ for each system $\omega$ we can choose the optimal system yielding maximum $\mathrm{U}$.

This concludes the discussion of various measures of effectiveness for comparison and choice of a source and a channel in persuading a given audience.. 


\section{GENERALIZATION}

We have presented a semi-Markovian model of the information processing and decision-making behavior of a consumer who is being persuaded by a communicator to make a desirable decision (i.e. to purchase a certain product) through a repeated communication of a message. The final objective was to yield some quantitative measures of effectiveness of a given message, source and channel and to consider the communicator's problem of optimally choosing the communication system.

Though the model was posed and analysed in the marketing context in order to yield a specific motivation, it seems clear that its broader implications can be interpreted in a general decision-making framework.

Consider a decision-maker who has to take an action a $€ \eta$ in face of an environment (state of the world) $e \varepsilon^{\rho}$ so as to yield a payoff $W(e, a)$, where 3 is the set of all available actions and $\&$ the set of all possible environments. The decision-maker's behavior is affected not only by the environment but alsc by the behavior of an external communicator who is trying to jnfluence the decision maker's choice of actions. (Thus the communicator's behavior together with the environment forms the set of uncontrollable variables from the decision-maker's viewpoint). The communicator chooses an input message $\mathrm{m} \in \mathrm{m}$, (where $m$ is the message space), a source $\mathrm{s} \in d$ (where $d$ is the set of all available sources), a channel $\mathrm{c} \in C$ (where $C$ is the set of all available channels) and an operating variable $\theta \in \Theta$ (where $\Theta$ is the set of available operating variables, e.g. values of scheduling variables such as $\mathrm{T}_{1}, \mathrm{~T}_{2}$, $\alpha$ etc.). In short, let $\omega=(\mathrm{m}, \mathrm{s}, \mathrm{c}$, ? $)$ be a communication system chosen from the set $\Omega=m \therefore \& \times C \times \Theta$. As a function of the environment $\mathrm{e} \epsilon \&$, and the decision- 
maker's action $a \hat{\varepsilon} \neg$ the communicator receives a payoff $R(e, a)$, and has to pay a cost of $\mathrm{C}(\mathrm{e}, \omega)$ for operating the system $\omega \in \Omega$ in environment e. For a given environment $e$ and the system $\omega=(m, s, c, \theta)$ the output message is $m^{\prime}=\gamma(e, \omega) \in m, m^{\prime}$ may be different from $m$ due to noise in the channel $c$, inaccuracies in coding at the source $s$ and operating characteristics $\theta$. This output message $\mathrm{m}^{\prime}$ together with the state of the world $e$ is further processed by the decision-maker's information processor $\eta$ to yield information $i=\eta\left(e, m^{\prime}\right) \in g$ (where $g$ is the set of all possible values of the information variables). The decision-maker uses a decision rule $\delta$ to yield an action $a=\delta(i) \in \neg$ on the basis of information $i$. Let $\Delta$ be the set of all decision rules. As a function of the environment $e$ and the action a the decisionmaker not only receives a payoff $W(e, a)$ but also incurs a cost $k(e, \eta, \delta)$ of operating the information processor $\eta$ and the decision rule $\delta$ in environment e.

If the environment set $\&$ has a probability measure $P$ defined (on its appropriately chosen $\sigma$-algebra), then the decision-maker's and the communicator's dual optionization problems could be stated in terms of the expected net returns with respect to $P$, as follows.

The communicator's problem is to choose a system $\omega=(m, s, c, \theta) \in M \times \& \times C \times \Theta=\Omega$, given the decision-maker's choice of an information processor $\eta$ and a decision rule $\delta$, so as to maximize $E[R(e, \delta(\eta(e, \gamma(e, \omega))))-C(e, \omega)]$. On the other hand, the decisionmaker's problem is to choose an information processor $\eta \in \eta$ and a decision rule $\delta \in \Delta$, given the communicator's choice of the system $\omega \in \Omega$, so as to 


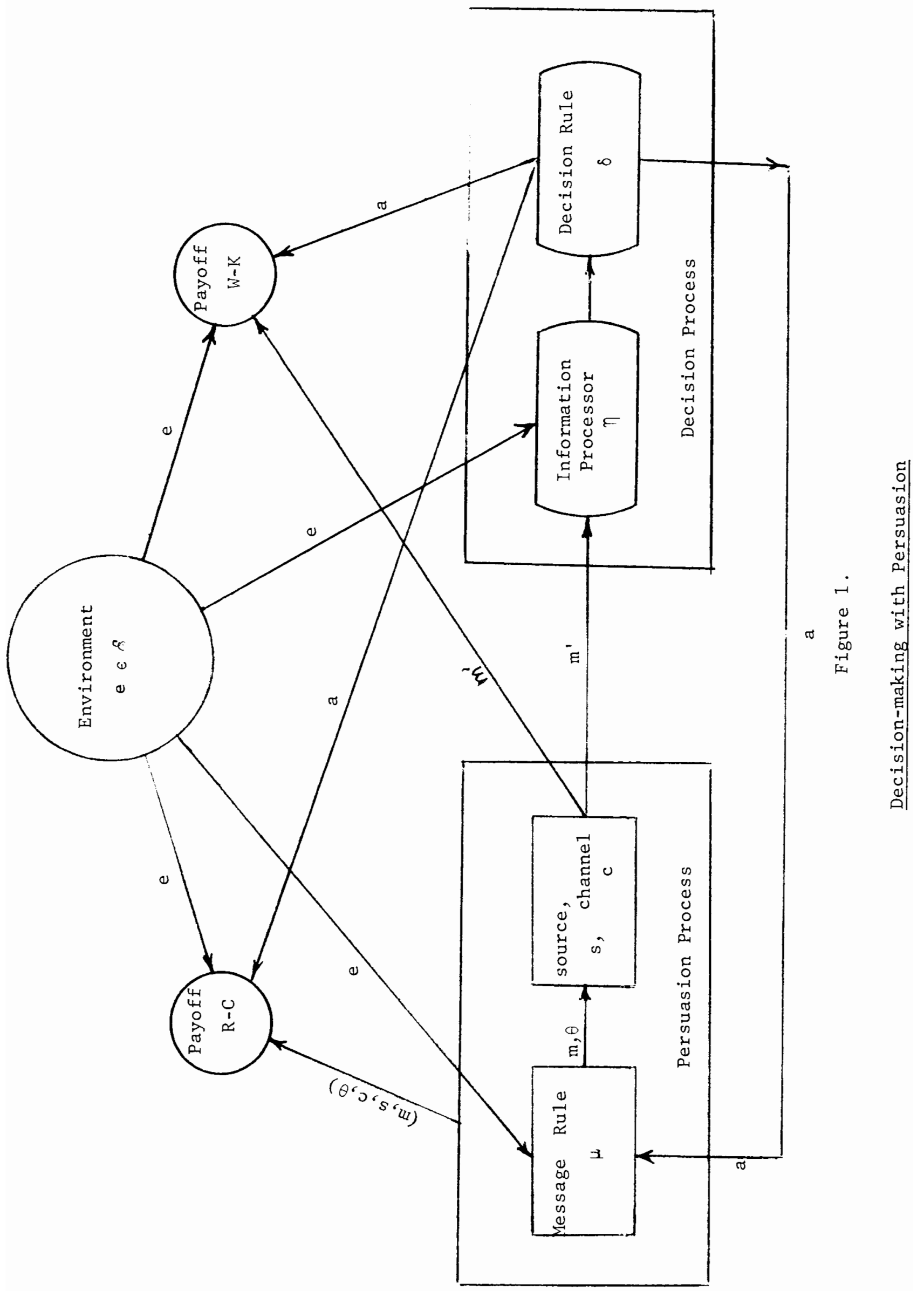


$\operatorname{maximize} \quad E[W(e, \delta(\eta(e, \gamma(e, \omega))))-K(e, \eta, \delta)]$

Thus the actions of each are included in the other's problem as being an uncontrollable variable (see Fig. 1).

Another application of this framework is to the analysis of decentralized planning for resource allocation in economic systems. Here we may view the planner as a communicator and each economic agent as a doricinn-maker. The

$$
\text { the environment e (e.g. technology, market. twicn }
$$

$\therefore a \cdot a$ (e.g. his production level or trades he offers $\therefore$ together

with the planning procedure $w$ (e.g. a mechanism for computing prices and incentives)

yield a message $m$ (e.g. prices, incentives, production requirements etc.).

The agent then processes this message $m$ together with his environment $e$ so as to yield a decision a. The utility of this process to the planner is $\mathrm{U}_{1}(\mathrm{e}, \mathrm{a}, \omega, \mathrm{m})$ while to the agent it is $\mathrm{I}_{2}(\mathrm{e}, \mathrm{a}, \omega, \mathrm{m})$, where $\mathrm{U}_{1}$ and $\mathrm{U}_{2}$ are, in general, different functions. The planner's persuasive behavior is reflected in his choosing the rules and incentives for following them as summarized in the message $m$ and the procedure $\omega$ so as to persuade each agent to select an action a closer to the one maximizing the expected value of $U_{1}$. On the other hand, given the planning procedure w, operating rules and incentive system $\mathrm{m}$, each economic agent's problem is to choose an action a maximizing the expected value of $\mathrm{U}_{2}$. We may call a procedure -message combination $(\omega, \mathrm{m})$ as being incentive-compatible if it results in each agent choosing an action a maximizing the expected values of both $U_{1}$ and $U_{2}$ simultaneously.

A natural framework for analyzing the problem of choosing optimal strategies for both then seems to be in the theory of stochastic games. 